\title{
Formation of Au-Silane Bonds
}

\author{
Shira Yochelis, ${ }^{1}$ Eran Katzir, ${ }^{1}$ Yoav Kalcheim, ${ }^{2}$ Vitaly Gutkin, ${ }^{3}$ \\ Oded Millo, ${ }^{2}$ and Yossi Paltiel ${ }^{1}$
}

\author{
${ }^{1}$ Department of Applied Physics, The Hebrew University of Jerusalem, Givat Ram, Jerusalem 91904, Israel \\ ${ }^{2}$ Racah Institute of Physics, The Hebrew University of Jerusalem, Givat Ram, Jerusalem 91904, Israel \\ ${ }^{3}$ The Unit for Nanocharacterization, The Harvey M. Krueger Family Center for Nanoscience and Nanotechnology, \\ The Hebrew University of Jerusalem, Jerusalem 91904, Israel
}

Correspondence should be addressed to Shira Yochelis, shirayo@cc.huji.ac.il

Received 8 January 2012; Revised 3 April 2012; Accepted 18 April 2012

Academic Editor: Ramón Antonio Zárate

Copyright $\odot 2012$ Shira Yochelis et al. This is an open access article distributed under the Creative Commons Attribution License, which permits unrestricted use, distribution, and reproduction in any medium, provided the original work is properly cited.

\begin{abstract}
Many intriguing aspects of molecular electronics are attributed to organic-inorganic interactions, yet charge transfer through such junctions still requires fundamental study. Recently, there is a growing interest in anchoring groups, which considered dominating the charge transport. With this respect, we choose to investigate self-assembly of disilane molecules sandwiched between gold surface and gold nanoparticles. These assemblies are found to exhibit covalent bonds not only between the anchoring Si groups and the gold surfaces but also in plane crosslinks that increase the monolayer stability. Finally, using scanning tunneling spectroscopy we demonstrate that the disilane molecules provide strong electrical coupling between the Au nanoparticles and a superconductor substrate.
\end{abstract}

\section{Introduction}

Organic-inorganic complexes bring many intriguing features to nanoscale phenomena and in particular to molecular electronics. Some of the attractive applications include organic light-emitting devices $[1,2]$ (OLEDs), memory devices, field-effect transistors (FETs), solar cells, and developing single-molecule and single-electron devices [3-6]. Consequently, for realizing molecular devices, it is crucial to understand how to exploit electron transport through organic molecules [7-9], metal passivation and corrosion [10], and surfaces' wetting $[11,12]$.

One of the key matters in molecular electronics is associated with charge transport through the molecule and the corresponding electrode-molecule-electrode junctions that is playing a critical role in the transport of the electron through the molecule $[13,14]$. The influencing factors in these junctions were illustrated by a systematic study of anchoring groups which affect the single-molecule conductance [15]. Chen et al. used alkanes terminated with different anchoring groups bound to gold electrodes as a model system and showed tight correlation to the bond strength. For example, $-\mathrm{SH}$ is known to form a strong $\mathrm{S}-\mathrm{Au}$ covalent bonds, $-\mathrm{NH}_{2}$-terminated alkanes are described by a weak covalent binding $[13,16]$, while $\mathrm{COOH}$ is believed to exhibit partially ionic and coordinated interactions [17]. A contact resistance was measured to confirm high sensitivity to the anchoring group and was found to vary in the order of $\mathrm{Au}-\mathrm{S}>\mathrm{Au}-\mathrm{NH}_{2}>\mathrm{Au}-\mathrm{COOH}$. The phenomenon was attributed to different electronic coupling efficiencies provided by the different anchoring groups between the alkane and the electrodes. Zotti et al. presented a combined experimental and theoretical study revealing the influence of the anchoring group on electronic transport through singlemolecule junction [18]. Transport experiments through tolane molecules attached to gold electrodes via thiol-, nitro-, and cyano- anchoring groups were performed and were fitted to a single-level tunneling model, which allowed extracting the position and broadening of the molecular levels that dominate the transport. Their calculation revealed that the anchoring groups control the nature of the conduction according to their donor or acceptor character. Recently, an analysis of the charge transport through single-molecule junction was performed to define the influence of molecular end groups for increasing electrode separation [19]. Inelastic electron tunneling spectroscopy results demonstrated that 
amino-ended molecules bind only weakly to $\mathrm{Au}$, in contrast to thiol-ended bond ones. It was explained that the strong $\mathrm{Au}$-thiol bond can cause a major mechanical deformation of the Au electrode. Other studies considered the dependence of the molecule junction conductance on the molecule conformation. It was shown that, for a series of seven biphenyl molecules with different ring substitutions that alter the twist angle of the molecule, the conductance for the series decreased with increasing the twist angle [20]. In another study the molecular conductance was measured by forming and breaking Au point contacts in the presence of amino-terminated molecules using modified scanning tunneling microscope (STM) [21]. It was found that the conductance of such an amino-Au bond does not vary significantly from junction to junction because of isotropy of the $\mathrm{N}$ lone pair to Au electronic coupling across the range of metal-molecule configurations. Such dependences are critical when considering development of devices for nanotechnological applications. Therefore, to understand the fundamental interface interactions and the various aspects of organized molecules on different surfaces via different anchoring groups is of utmost importance.

Another reason for the chemistry of gold surface to be extensively investigated is the important role that the surface of gold exhibits in catalysis [22, 23] and nanotechnology [24]. The covalent bond of gold is in the core of ligandprotected gold nanoparticles (NPs) and heterogeneous catalysis of organo-gold hybrids. Due to the importance of such systems, it is essential to extend the study of adsorption on gold surface to variety of molecules and linking groups. Gold surface adsorption was investigated so far mainly for thiol [25, 26], amine [27-29], phosphine [30, 31], and cyanide [24] head groups. We show here that selfassembly of alkanes with silane head groups gives $\mathrm{Au}-\mathrm{Si}-\mathrm{Au}$ sandwich systems that exhibits stable in plane polymerized monolayers. Consequently, this paper presents a new hybrid system sensitive to the coupling and therefore to charge and energy transfer through the organic molecules' monolayer. Up to now, reports on organic molecules with silane head groups adsorbed to gold surfaces were limited and the nature of the corresponding bond type is still controversial.

The $\mathrm{Si}-\mathrm{Au}$ bond was presented previously by several groups. Fundamental study performed in ultrahigh pressure proved the existence of the $\mathrm{Si}-\mathrm{Au}$ bond [32]. The authors characterized the bond formation by reflection absorption infrared spectroscopy (RAIRS) and X-ray photoelectron spectroscopy (XPS). The RAIRS results demonstrated that the prominent $\mathrm{Si}-\mathrm{H}$ bond seen for the solution of the octylsilane molecule was absent for the silane-based layers. Moreover, XPS data exhibited reduced $\mathrm{Si} 2 \mathrm{p}_{3 / 2}$ binding energy at $99.8 \mathrm{eV}$ supported the $\mathrm{Si}-\mathrm{Au}$ bond. The bonding geometry was proposed to display $\mathrm{Si}$ atom that forms three covalent bonds to the gold surface. A different study demonstrating self-assembly from tetrahydrofuran solution of porphyrin oligo(phenyleneethynylene trimethylsilylethynyl) (P-OPE-silane) was carried out by Watcharinyanon et al [33]. They reported high-resolution XPS binding energy data for $\mathrm{Si} 2 \mathrm{p}_{3 / 2}$ at $101.9 \mathrm{eV}$. The authors offered that the higher position of the Si binding energy peak was due to the fact that not all $\mathrm{Si}-\mathrm{CH}_{3}$ bonds were cleaved upon the film formation. Combining with their near-edge X-ray absorption fine structure spectroscopy results, the authors suggested that the self-assembled monolayer (SAM) had a poorly defined orientational order. An earlier work of self-assembled trichloro(octadecyl)silane (OTS) performed by Sabatani et al. $[27,34]$ exhibited electrochemical and reflection-absorption Fourier transform infrared measurements displayed well-defined crosslinked monolayer on gold electrode though not defect-free. In a further study, Olson et al. [35] showed that gold colloids were protected from exposure to solution-phase species upon surface modification by silane. Surface-enhanced Raman scattering (SERS) measurements exhibited enhancement of the substrate signal for immobilized gold colloids coated with wellpacked highly ordered (n-octadecyl)trimethoxysilane with high reproducibility even when exposed to solutes. In their case it was stated that this monolayer eliminates the chemical or charge transfer contribution to the SERS mechanism due to the monolayer protection. In agreement with the previous results we present XPS results that demonstrate a reduced $\mathrm{Si}$ which we relate to $\mathrm{Si}-\mathrm{Au}$ covalent bond. However, this reduced Si peak does not appear for all the studied molecules. To ensure that the $\mathrm{Si}-\mathrm{Au}$ bond is universal and exists also for the specific studied molecules, we performed additional coupling measurements between gold NPs through the organic monolayer to a type II superconductor (serving as a sensitive sensor to charge or energy transfer). The results show that the superconductor properties are modified upon the adsorption of gold NPs on top of the molecular layers indicating that an efficient charge transfer mechanism exists through the Si-Au bond.

\section{Materials and Methods}

Self-assembly from a solution is a simple, fast, and reproducible method for producing organized monolayers suitable for molecular electronics. For our study we used three types of silane molecules (Figure 1) that were adsorbed to gold or Nb surfaces, each forming a different type of goldmolecule hybrid system. In all of the different cases the Si$\mathrm{Au}$ bond formation was identified. One system SAM consists of 2-methylene-(1,3-propanediyl)bis(trichlorosilane) (MPdS) or 1,6-bis(trichlorosilyl)hexane (Hexa) molecules sandwiched between a gold or Nb surface and gold NPs chemically attached to them, as will be detailed below. The other system consists of OTS molecule with only one silane head group assembled on gold or $\mathrm{Nb}$ surface, providing no linking to the Au NPs deposited on top of them. The OTS molecule was chosen to monitor the Si$\mathrm{Au}$ bond formation and to serve as a reference to the MPdS and Hexa that exhibit a bridge between two different $\mathrm{Au}$ surfaces. Chemisorbed monolayers of MPdS, Hexa, and OTS were formed by immersing evaporated gold $(\mathrm{Cr}: \mathrm{Au}$ $(10: 100 \mathrm{~nm})$ ) or sputtered $\mathrm{Nb}$ films (on Al film on top of Si wafer) in diluted solutions of $1 \mathrm{mM}$ organic molecules in bicyclohexyl solution or OTS in toluene solution for $2 \mathrm{~min}$ followed by sonication in toluene for $1 \mathrm{~min}$. This procedure 


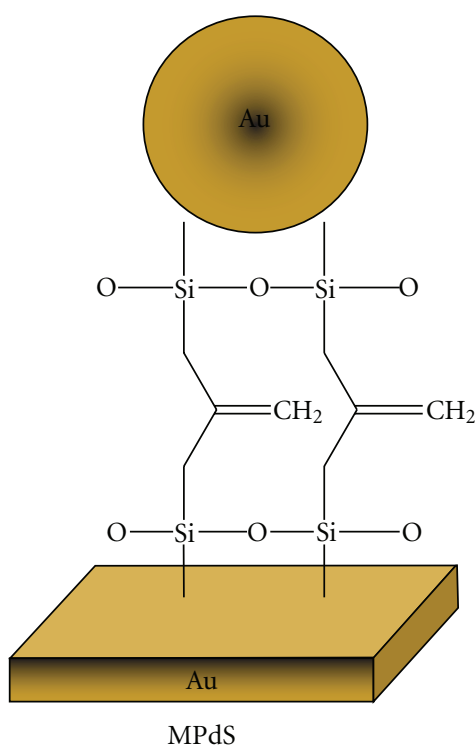

(a)

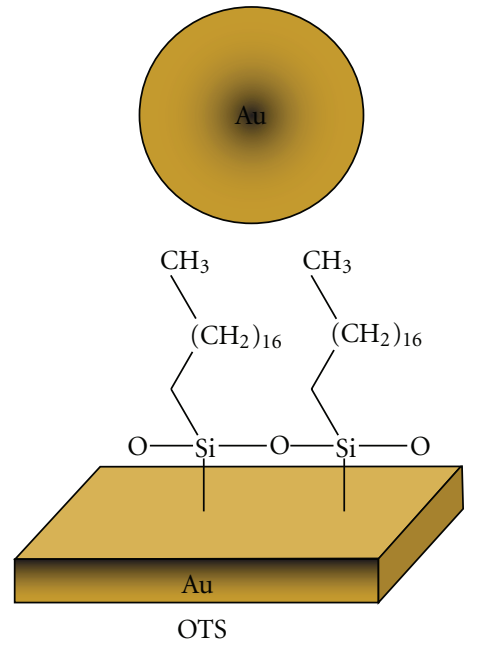

(b)

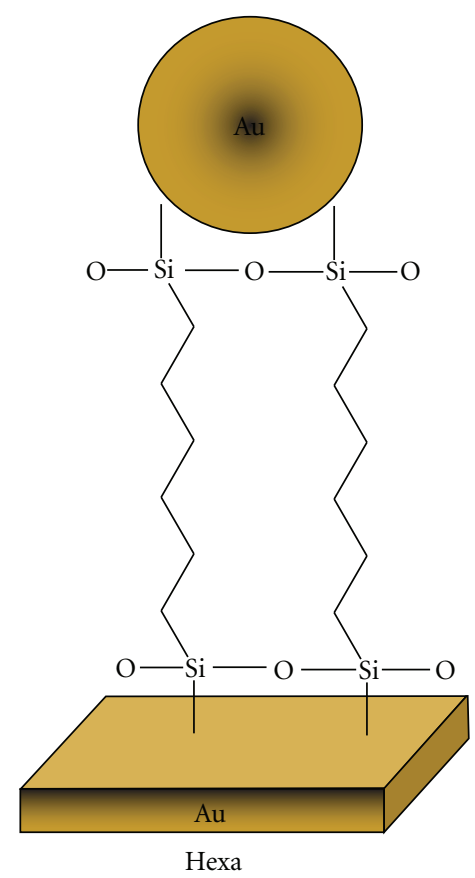

(c)

FIGURE 1: Schematic description of the organic molecules that were used for the SAM on Au or Nb substrate.

was repeated twice, followed by dipping the samples in $10 \mathrm{~nm}$ trisodium citrate hydrated gold solution for 24 hours or in the case of Hexa dipped in dodecanethiol functionalized gold nanoparticles in toluene. The samples were then washed with water or toluene and dried by nitrogen. The reactions were carried out under nitrogen environment, and the solvents chosen were anhydrous. The resulting SAMs were characterized by XPS, contact angle measurements, scanning electron microscopy (SEM), Woallam alpha-SE ellipsometry, and scanning tunneling microscopy (STM). STM tunneling spectra were collected for the systems that were lacking the XPS contact confirmation. Finally, measurements of VI characteristic of a $\mathrm{Nb}$ superconducting film coupled by the $\mathrm{Si}-\mathrm{Au}$ bond to gold NPs demonstrate enhancement of the critical current, implying charge coupling between the $\mathrm{Au}$ dots and the $\mathrm{Nb}$ film.

The XPS measurements were performed with a Kratos AXIS Ultra X-ray photoelectron spectrometer (Kratos Analytical Ltd., Manchester, UK). Spectra were acquired using the $\mathrm{Al}-\mathrm{K}_{\alpha}$ monochromatic X-ray source $(1,486.7 \mathrm{eV})$. Sample take-off angle was $90^{\circ}$ (relative to the analyzer). The vacuum pressure in the analyzing chamber maintained to $2 \cdot 10^{-9}$ Torr. High-resolution XPS spectra were collected for $\mathrm{C} 1 \mathrm{~s}, \mathrm{O} 1 \mathrm{~s}, \mathrm{Si} 2 \mathrm{p}$, and Au $4 \mathrm{f}$ peaks with pass energy $20 \mathrm{eV}$ and $0.1 \mathrm{eV}$ stepsize. The XPS binding energies were calibrated with respect to the peak position of the $C$ s peak as $285.0 \mathrm{eV}$. Data analyses were done using the Kratos Vision data reducing processing software (Kratos Analytical Ltd.) and Casa XPS (Casa Software Ltd.). From the results C 1s (I) characteristic binding energy of $285.0 \mathrm{eV}$ is corresponding to the hydrocarbon chains $\left(\mathrm{CH}_{x}\right)$ of the SAMs. In addition, two other peaks C 1s (II) and C 1s (III) around $286.6 \mathrm{eV}$ and $289.7 \mathrm{eV}$ (for Au substrate) appear at higher binding energy indicating the exposure to air. The peaks are related to C$\mathrm{O}$ bonds and $\mathrm{C}=\mathrm{O}$ probably coming from oxidation. The monolayers' extra peak appears only for the $\mathrm{C}-\mathrm{O}$ bond, at $286.6 \mathrm{eV}$. This suggests less oxidation of the samples which correlates with keeping the samples under nitrogen.

Using the XPS measurements, it is possible to calculate the thickness of the assembled layers. We have done so using the standard attenuation relations of the photoelectrons emerging from different sample depths. The thickness calculation is based on the Briggs et al. method and others [36-38].

For the Au substrate, the overlay thickness $d$ (nm) expressed as

$$
d=\lambda_{C} \sin \theta \ln \left(\frac{N_{\mathrm{Au}} \lambda_{\mathrm{Au}} I_{o}}{N_{\mathrm{C}} \lambda_{\mathrm{C}} I_{\mathrm{Au}}}+1\right),
$$

where $I_{\mathrm{Au}}$ and $I_{o}$ are the area intensities (area percentages) of $\mathrm{Au}$ and the overlayer peaks, respectively, (then $I_{o}=I_{\mathrm{C}}+I_{\mathrm{O}}+I_{\mathrm{Si}}$ are the intensities for carbon, oxygen, and silicon peaks), $\theta$ is the take-off angle (in our case $\sin \theta=1$ ), and $N_{\mathrm{Au}}$ and $N_{\mathrm{C}}$ are the volume densities. The inelastic mean free paths (IMFPs) parameters $\lambda_{\mathrm{C}}$ for the overlayer and $\lambda_{\mathrm{Au}}$ for the substrate were assumed as $3.3 \mathrm{~nm}$ and $2.5 \mathrm{~nm}$, respectively [36].

In addition to the XPS results, thickness measurements were performed also by ellipsometry. It was determined by the Cauchy model ( $\mathrm{An}=1.5$ and $\mathrm{Bn}=0.01$, as these are good default values for many organic films). 


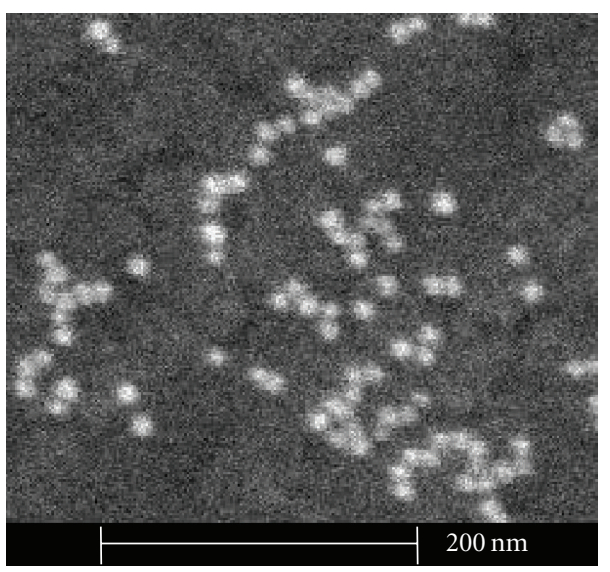

(a)

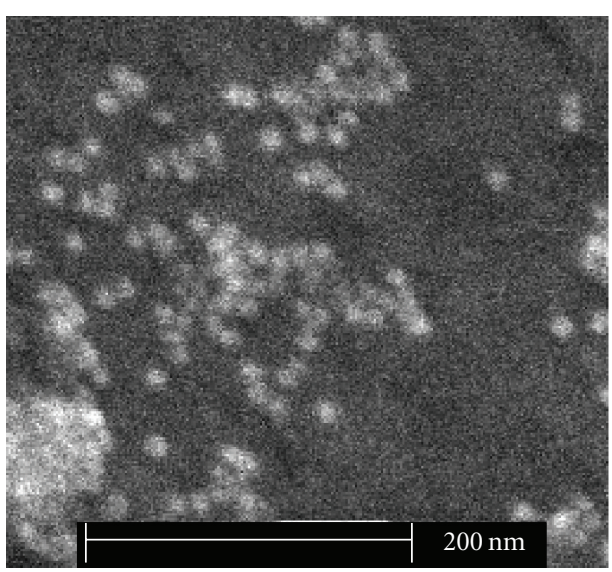

(b)

Figure 2: SEM images showing gold NPs (bright dots) adsorbed on top of an MPdS monolayer self-assembled on a gold film. (a) After adsorption, (b) after washing with acetone and ethanol under sonication and dipping in hot toluene.

TABLE 1: High-resolution XPS data: peak position $(\mathrm{eV})$ of $\mathrm{Si} 2 \mathrm{p}$ and $\mathrm{Au} 4 \mathrm{f}_{7 / 2}$ spectra.

\begin{tabular}{|c|c|c|c|c|c|}
\hline & $\mathrm{Si}$ & $\mathrm{Si}-\mathrm{O}$ & $\mathrm{Si}-\mathrm{OH}[2 \mathrm{p}(\mathrm{II})]$ & $\mathrm{Si}-\mathrm{Au}[2 \mathrm{p}(\mathrm{I})]$ & $\mathrm{Au}\left[4 \mathrm{f}_{7 / 2}\right]$ \\
\hline Binding energy (eV) [39] & 99.2 & $103.2-103.6$ & 103.5 & & 84.0 \\
\hline OTS & & $\mathrm{X}$ & $\mathrm{X}$ & 101.8 & 84.0 \\
\hline OTS (bulk*) & & $102.6[2 p(I)]$ & & & \\
\hline Hexa & & $102.7[2 \mathrm{p}(\mathrm{II})]$ & $\mathrm{X}$ & 101.5 & 84.0 \\
\hline MPdS & & $103[2 \mathrm{p}(\mathrm{I})]$ & $103.5[2 \mathrm{p}(\mathrm{II})]$ & $\mathrm{X}$ & 84.0 \\
\hline MPdS (bulk*) & & $102.9[2 \mathrm{p}(\mathrm{I})]$ & $104.1[2 \mathrm{p}(\mathrm{II})]$ & & \\
\hline Au substrate & & & & & 84.0 \\
\hline
\end{tabular}

Bulk—refers to an organic solution sample that was repeatedly dried under nitrogen stream on top of the gold surface.

\section{Results and Discussions}

The first different between the molecules types is reflected in the durability towards attempts to removed the adsorbed gold NPs. Thoroughly washing the MPdS samples with acetone, ethanol, and distilled water (DW) under sonication and hot toluene did not remove the gold NPs, as seen by SEM images (taken using Serion from FEI) presented in Figure 2. In contrast, $\mathrm{Au}$ NPs deposited on OTS were easily washed out.

The XPS results for MPdS, Hexa, and OTS SAMs on Au substrate are summarized in Table 1 . Based on our results and previous studies by Sabatani et al. [34], it is realistic to assume that the $\mathrm{SiCl}_{3}$ goes through oxidation to $\mathrm{Si}(\mathrm{OH})_{3}$ and then chemisorbed to the surface while presenting a stable polymerized in plane monolayer having $\mathrm{Si}-\mathrm{O}-\mathrm{Si}$ groups with defects in the monolayer as evident by $\mathrm{Si}-\mathrm{OH}$ free groups. Focusing on the different Si bonds, we find three types of bonds, two oxidized Si: $\mathrm{Si}-\mathrm{O}, \mathrm{Si}-\mathrm{OH}$, and one reduced $\mathrm{Si}$. We show that, for the Hexa and OTS monolayers, the results exhibit reduced $\mathrm{Si}$ that is argued as the $\mathrm{Si}-\mathrm{Au}$ bond (as no other reasonable bond is found in that specific energy). However, for the MPdS monolayer, we find only oxidized Si.

For the Hexa and OTS monolayers, we find a reduced Si 2 p (I) peak: at $101.8 \mathrm{eV}$ for the OTS (Figure 3(a)) and at $101.5 \mathrm{eV}$ (Figure 3(b)) for the Hexa (the $\mathrm{Si}^{0}$ doublet is found at $99.2 \mathrm{eV}$ and $99.8 \mathrm{eV}$ binding energies). The results suggest that this Si exhibits $\mathrm{Si}-\mathrm{Au}$ bond keeping in mind the monolayer remained on the surface also after sonication in toluene. Comparing it to the OTS bulk sample (adding repeatedly the organic molecule solution and drying under nitrogen stream on top of the gold surface) demonstrating Si 2p (I) at $102.6 \mathrm{eV}$, it seems that the $\mathrm{Si}-\mathrm{Au}$ binding energy peak is missing. This indicates that the OTS and Hexa monolayers exhibit $\mathrm{Si}-\mathrm{Au}$ bond in a good agreement to previous study that presented reduced Si peak at $101.9 \mathrm{eV}$ [33]. The Hexa monolayer exhibits also an oxidized Si 2p (II) at $102.7 \mathrm{eV}$. It is reasonable to assume that the organic assembly appears as inplane polymerized monolayer composed of Si-O-Si bridges between the molecules [39], as the silane chloride exchanges the chloride ions by hydroxyl groups to have a condensation of the $\mathrm{Si}-\mathrm{OH}$ free groups.

In contrast, the MPdS monolayer shows two binding energy positions: for Si $2 \mathrm{p}$ (I) at $103 \mathrm{eV}$ and for Si $2 \mathrm{p}$ (II) at $103.5 \mathrm{eV}$ (Figure 3(c)). This involves two bond types. Both of the $\mathrm{Si}$ are oxidized as for the high binding energies. XPS and ellipsometry thickness measurements for the MPdS and OTS monolayers are given in Table 2. A comparison between the different thickness measurements and the estimated length for the MPdS molecule indicates for a multilayer assembly, assumed a continuous film. For rough calculation points it is composed of about seven layers cross-linked by $\mathrm{Si}-\mathrm{O}-\mathrm{Si}$ 


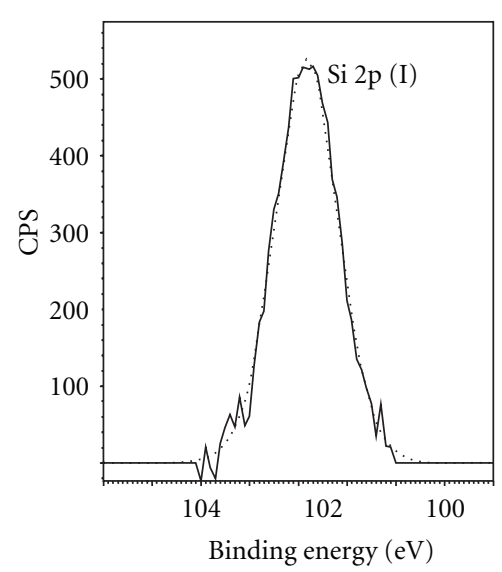

(a)

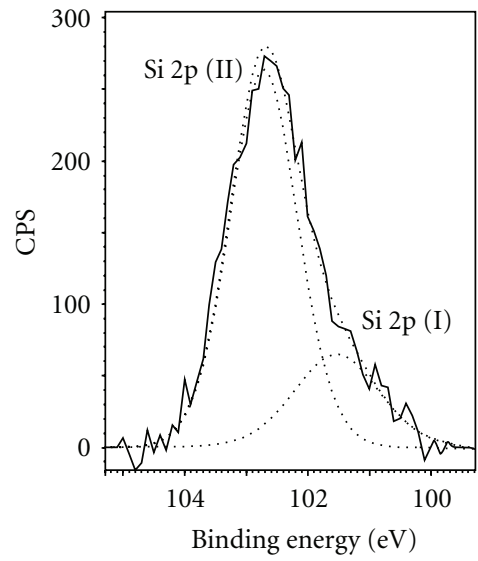

(b)

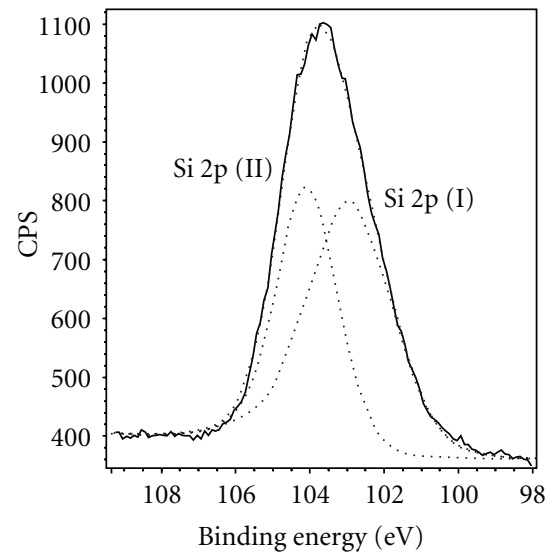

(c)

FIgURE 3: High-resolution XPS spectra of Si 2p levels for (a) OTS, (b) Hexa, and (c) MPdS samples.

TABLE 2: Thickness measurements.

\begin{tabular}{lcc}
\hline & MPdS & OTS \\
\hline XPS $(\mathrm{nm})$ & 4.7 & 2.6 \\
Ellipsometry $(\mathrm{nm})$ & $4.5 \pm 0.06$ & $2.5-2.8$ \\
Estimated length $(\mathrm{nm})$ & 0.6 & 2.6 \\
\hline
\end{tabular}

bonds. This may explain the absence of the reduced $\mathrm{Si}-\mathrm{Au}$ bond in the XPS spectra as it is in a very small amount compared to the oxidized Si. The first Si $2 p$ (I) is probably attributed to the Si-O-Si species. The second Si 2p (II) peak at $103.5 \mathrm{eV}$ demonstrates the existence of $\mathrm{Si}-\mathrm{OH}$ free groups which indicates that the crosslinking in plane is not complete. As illustrated by the XPS and ellipsometry thickness results, the OTS is arranged as one layer-thick monolayer, normal to the surface.

Nevertheless, as mentioned before, the Au NPs attached to the monolayer stay adherent to the layer also after roughly washing the samples under sonication in acetone and ethanol and dipping in hot toluene (see Figure 2). We also examined the bulk sample of MPdS prepared by adding repeatedly the organic molecule solution and drying under nitrogen stream on top of the gold surface. The results exhibit two oxidized Si bonds at $102.9 \mathrm{eV}$ and $104.1 \mathrm{eV}$ as in the monolayer. As mentioned before, the first corresponds to $\mathrm{Si}-\mathrm{O}-\mathrm{Si}$ bond and the second to $\mathrm{Si}-\mathrm{OH}$ groups that did not cross-link to $\mathrm{Si}-$ $\mathrm{O}-\mathrm{Si}$ bonds. The gold amount (atomic percent) reduced by 6 as a result of masking it by large amount of the organic molecule. In addition, we looked at Au take-off angle in order to examine whether it is possible to gain some information from the surface $\mathrm{Au}$ atoms that are bonded to the silane group. The results exhibit very stable $83.99-83.97 \mathrm{eV}$ signals at angles between 0 and $60^{\circ}$ meaning it is not possible to measure the oxidized $\mathrm{Au}$ as a result of the binding to the silane group. We believe that the difficulty is arising from the negligible amount of surface $\mathrm{Au}$ atoms that are oxidized compared to the bulk Au substrate.
Contact angle of the SAMs shows an angle of $70^{\circ}$ for the MPdS SAM and $111^{\circ}$ for the OTS. By the results of the OTS, it appears as hydrophobic SAM which indicates the formation of SAMs on the surface. Regarding the MPdS layer, the angle is too small to imply fully covered SAM surface. This may result from the discontinuous layer evidenced by the XPS results which demonstrates free $\mathrm{Si}-\mathrm{OH}$ groups.

Comparing our results to previously reported results using different techniques of film formation, it was shown that $\mathrm{Au}-\mathrm{Si}$ bond appears at $99.8 \mathrm{eV}$ for tricoordinated $\mathrm{Au}$, and $101.9 \mathrm{eV}$ for lower coordinated $\mathrm{Au}[32,33]$. These results were explained by both theoretical and practical studies claiming that Au prefers dicoordinated bonding [34]. As was mentioned before, we believe that in our case it is realistic to assume that the $\mathrm{SiCl}_{3}$ goes through oxidation to $\mathrm{Si}(\mathrm{OH})_{3}$ and then chemisorbed to the surface while presenting a stable polymerized in-plane monolayer with defects as evident by the $\mathrm{Si}-\mathrm{OH}$ free groups. This was supported by Sabatani et al. study [34] for the OTS measured by electrochemical and RAFTIR measurements. We find that the silane anchoring group is attached directly to the gold surface from the XPS results having reduced Si $2 \mathrm{p}$.

The above explanation should also be true for the MPdS molecules; however, in this case we did not find a reduce Si $2 p$ (I) peak. Therefore, to further examine whether covalent bond exists also for the MPdS molecule, we measured electrical coupling between the Au NPs and a superconductor through the organic layers. For this we used scanning tunneling spectroscopy and transport measurements. As detailed below, strong coupling and efficient charge transfer were found to exist between the $\mathrm{Nb}$ film and the Au NPs. This behavior is manifested both in our scanning tunneling spectroscopy measurements, monitoring a modified density of states, and in transport measurements, revealing significant changes in the critical currents. For these experiments, the $\mathrm{Nb}$ films were sputtered on a $100 \mathrm{~nm}$ aluminum film on silicon wafers. As detailed elsewhere [40], the transition temperature, $T_{C}$, changed noticeably when using molecules having two silane end groups, whereas 


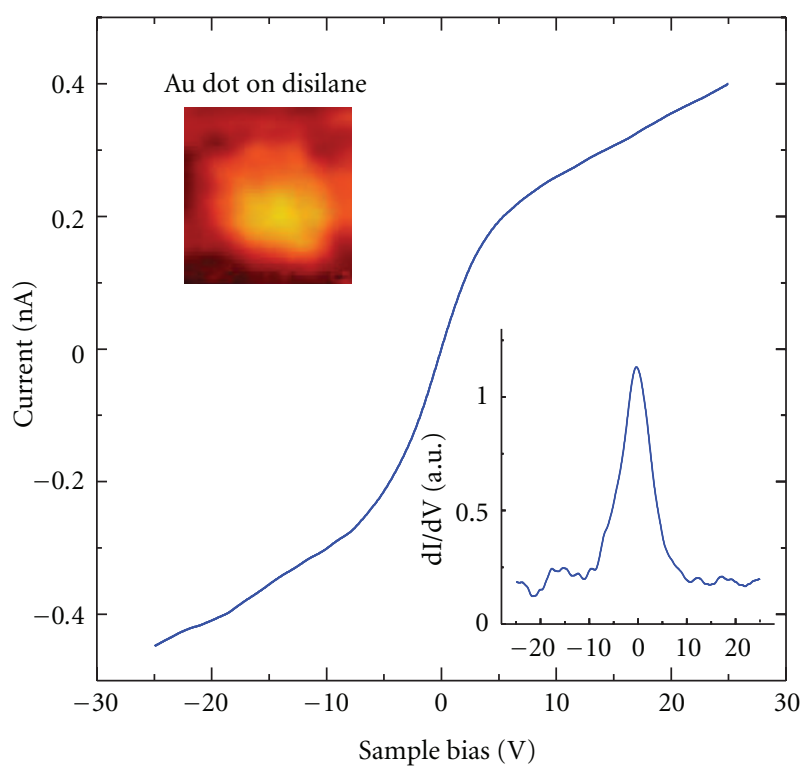

(a)

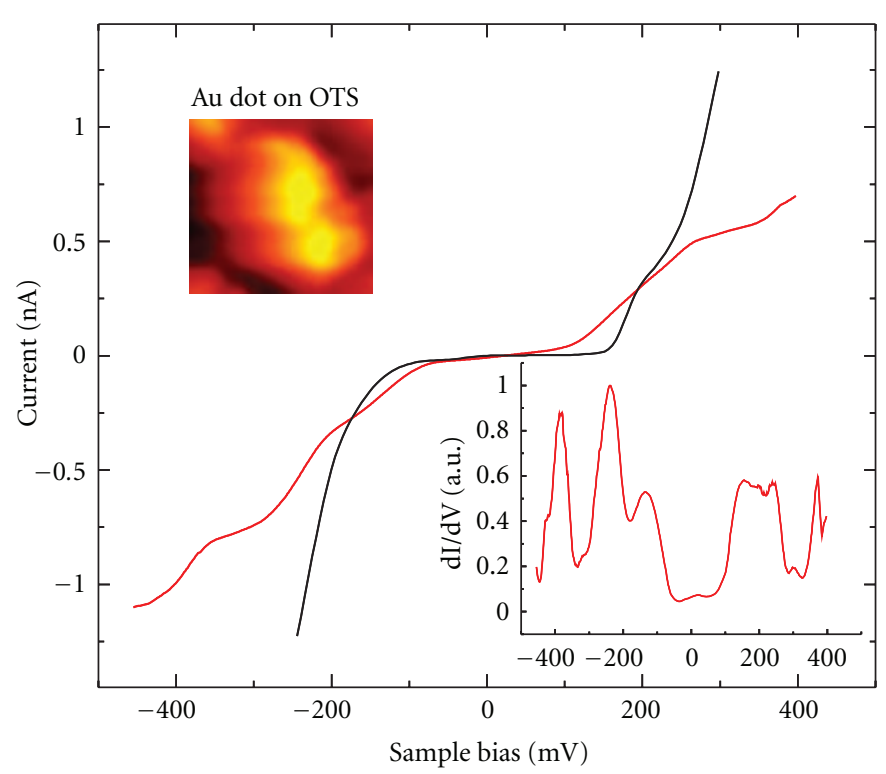

(b)

FIgURE 4: Tunneling I-V characteristics at $4.2 \mathrm{~K}$ acquired on single $5 \mathrm{~nm}$ dia. Au NPs deposited on two different molecular layers selfassembled on a $150 \mathrm{~nm}$ superconducting Nb film. (a) On MPdS (di-Silane), showing enhanced zero-bias conductance that reflects strong electrical coupling to the $\mathrm{Nb}$ film. Inset: the corresponding $\mathrm{dI} / \mathrm{dV}-\mathrm{V}$ spectrum. (b) On OTS, showing single-electron tunneling effects that indicates weak electrical coupling. Two curves are presented, taken with different STM settings. The black curve shows a Coulomb blockade, which is removed in the red curve that exhibits a clear Coulomb staircase. Inset: derivative spectrum of the red curve. The voltage-current settings were (a) $V_{\text {set }}=-10 \mathrm{mV} ; I_{\text {set }}=-0.3 \mathrm{nA}$, (b) $V_{\text {set }}=-200 \mathrm{mV} ; I_{\text {set }}=-0.5$ and $-0.35 \mathrm{nA}$, for the black and red curves, respectively.

no change was observed for OTS, implying good electrical contact and thus chemical linking only in the former case. Tunneling spectra acquired using STM on the Au dots reveal consistently different behaviors for Au NPs that are chemically attached to $\mathrm{Nb}$ by MPdS compared to the $\mathrm{Au}$ NPs that are adsorbed to the Nb. Figure 4 presents tunneling $\mathrm{I}-\mathrm{V}$ characteristics measured at $4.2 \mathrm{~K}$ on single Au NPs deposited either on top of MPdS (a) or OTS (b) monolayers self-assembled on $\mathrm{Nb}$. The I-V curves acquired for MPdS (Figure 4(a)) show enhanced zero-bias conductance, which clearly manifests itself as a pronounced peak in the tunneling $\mathrm{dI} / \mathrm{dV}$ versus $\mathrm{V}$ spectra (proportional to the local density of states) [41] presented in the inset. It is important to note that the existence of this zero-bias conductance peak was independent of the STM current and voltage settings (before disabling the feedback loop for spectrum acquisition) and disappeared above $T_{C}$. As detailed in [41], this peak is associated with coupling between electronic states in the $\mathrm{Nb}$ and $\mathrm{Au}$ dots, resulting in $T_{C}$ modification. Such coupling points to the formation of $\mathrm{Au}-\mathrm{Si}$ chemical bonding. In contrast to these results, the tunneling spectra measured on Au NPs deposited on OTS have never shown enhanced zero-bias conductance and rather exhibited single-electron charging effects (Coulomb blockade and staircase) [42], indicating weak electrical coupling to the $\mathrm{Nb}$ film, even though the thickness of the OTS layer is smaller than that of the MPdS (we note in passing that, far enough from the $\mathrm{NPs}$, the spectra exhibited the superconducting gap of $\mathrm{Nb}$ ). Figure 4(b) demonstrates this effect. The blue curve exhibits a region around zero bias where current was immeasurable (the Coulomb blockade), followed by a step-like feature that can be associated with the addition of a single electron to the dot (staircase). Upon changing the STM current-voltage setting, an effect similar to changing the gate voltage in single-electron tunneling devices [36, 42, 43], the Coulomb blockade, can be suppressed, resulting in finite conductance at zero bias. This effect is depicted by the red curve in Figure 4(b) and by its derivative, presented in the inset, in which the staircase (at higher-bias voltages) is more clearly evident. Although the I-V curves presented in Figure 4(b) do not exactly conform to the "text book case" single-electron tunneling characteristics excepted for metallic dots [43, 44], possibly due to the effect of the OTS molecules, the data clearly demonstrate that the coupling between the Au NPs and the $\mathrm{Nb}$ substrate is weak, indicating that they are only physisorbed on the OTS layer, in contrast to the case of MPdS. The STM results thus support our picture that MPdS indeed provides good covalent linking between the Au NPs and the substrate, allowing for charge transfer through the organic molecule.

Lastly, when gold NPs are in proximity to a superconductor below $T_{C}$, a proximity influence is expected to take place. This proximity effect is induced by the Andreev reflection [44] and therefore stimulates charge transfer through the organic molecules. Coupling our hybrid layers to a type II superconductor enables us to measure the fingerprint of this charge or energy transfer. With the change of the gold NP state, pinning or antipinning centers are induced to the system thus changing the superconductor critical current [45]. The superconductor in this case acts as a sensitive probe 


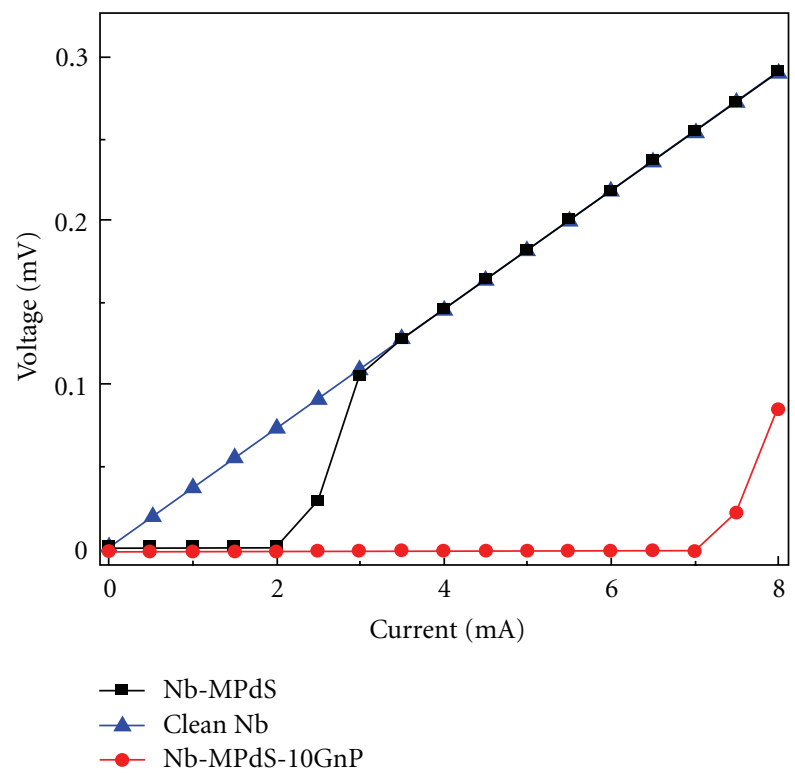

FIGURE 5: V-I characteristics of $150 \mathrm{~nm} \mathrm{Nb}$ superconductor film at $8 \mathrm{~K}$, just below the critical temperature for superconductor transition. The black line presents data corresponding to clean $\mathrm{Nb}$ film, where the critical current (onset of voltage drop) is around $2 \mathrm{~mA}$. The blue line was measured on the same $\mathrm{Nb}$ film after MPdS adsorption, showing that the critical current is suppressed. The red line presents the situation after $10 \mathrm{~nm}$ dia. gold NPs were deposited onto the MPdS, significantly enhancing the critical current to around $8 \mathrm{~mA}$.

for charge or energy transfer through the organic matter. Indeed, we observed an increase in the critical current of a $\mathrm{Nb}$ superconducting film when gold NPs are deposited on top of the $4 \mathrm{~nm}$ thick MPdS layer, as demonstrated by Figure 5 . This effect is quite remarkable, since the adsorbed MPdS monolayer alone destroys the film's superconductivity due to surface effects [46] and the critical current goes to zero (blue curve), while, by adsorbing $10 \mathrm{~nm}$ dia. Au NPs, the critical current increases from $2 \mathrm{~mA}$ for the bare film (black curve) to around $8 \mathrm{~mA}$ (red curve) with the NPs. These results show that the strong crosslinking of the disilane molecules creates an efficient network for charge transfer even at distances which were assumed to be too large for tunneling effects.

\section{Conclusions}

The present work demonstrates that, despite the gold nobility, it may be activated by silane groups to form highly stable and crosslinked organized monolayer even not fully covers the gold surface. Moreover, the organized monolayer networks maintain strong coupling to the substrate even at 3-4 $\mathrm{nm}$ distances. We believe that our results provide a paramount step towards understanding the chemical mechanism of the Au-Si bond formation. From the technological point of view, we have showed that silane-based SAMs are viable potential components for stable hybrid organic Au devices.

\section{Acknowledgments}

Special thanks are given to Dr. Nadav Katz from the Hebrew university who has grown the superconducting $\mathrm{Nb}$ clean films. The authors would like to acknowledge the support of the Peter Brojde Center for Innovative Engineering and Computer Science. O. Millo thanks support from the USIsrael BSF foundation, the German-Israel DIP program, and the Harry de Jur Chair in Applied Science.

\section{References}

[1] B. Abderraouf, D. Ban, H. Luo et al., "InAsSb based midinfrared optical upconversion devices operable at thermoelectric temperatures," IEE Electronics Letters, vol. 44, no. 4, pp. 312-313, 2008.

[2] P. K. H. Ho, J. I. S. Kim, J. H. Burroughes et al., "Molecularscale interface engineering for polymer light-emitting diodes," Nature, vol. 404, no. 6777, pp. 481-484, 2000.

[3] M. A. Kastner, "Artificial atoms," Physics Today, vol. 46, no. 1, pp. 24-31, 1993.

[4] M. H. Dvoret, D. Esteve, and C. Urbina, "Single-electron transferin metalic nanostructures," Nature, vol. 360, pp. 547$553,1992$.

[5] J. M. Tour, "Molecular electronics. Synthesis and testing of components," Accounts of Chemical Research, vol. 33, no. 11, pp. 791-804, 2000.

[6] J. Jortner and M. Ratner, Molecular Electronics, Blackwell, London, UK, 1997.

[7] Y. Paltiel, A. Aharoni, U. Banin, O. Neuman, and R. Naaman, "Self-assembling of InAs nanocrystals on GaAs: the effect of electronic coupling and embedded gold nanoparticles on the photoluminescence," Applied Physics Letters, vol. 89, no. 3, Article ID 033108, 2006.

[8] T. Aqua, R. Naaman, A. Aharoni, U. Banin, and Y. Paltiel, "Hybrid nanocrystals-organic-semiconductor light sensor," Applied Physics Letters, vol. 92, no. 22, Article ID 223112, 2008.

[9] M. A. Rampi and G. M. Whitesides, "A versatile experimental approach for understanding electron transport through organic materials," Chemical Physics, vol. 281, no. 2-3, pp. 373-391, 2002.

[10] F. P. Zamborini and R. M. Crooks, "Corrosion passivation of gold by n-alkanethiol self-assembled monolayers: effect of chain length and end group," Langmuir, vol. 14, no. 12, pp. 3279-3286, 1998.

[11] P. E. Laibinis, R. G. Nuzzo, and G. M. Whitesides, "Structure of monolayers formed by coadsorption of two n-alkanethiols of different chain lengths on gold and its relation to wetting," Journal of Physical Chemistry, vol. 96, no. 12, pp. 5097-5105, 1992.

[12] P. E. Laibinis, G. M. Whitesides, D. L. Allara, V. T. Tao, A. N. Parikh, and R. G. Nuzzo, "Comparison of the structures and wetting properties of self-assembled monolayers of nalkanethiols on the coinage metal surfaces, $\mathrm{Cu}, \mathrm{Ag}, \mathrm{Au}$," Journal of the American Chemical Society, vol. 113, no. 19, pp. 7152-7167, 1991.

[13] H. B. Akkerman, P. W. M. Blom, D. M. De Leeuw, and B. De Boer, "Towards molecular electronics with large-area molecular junctions," Nature, vol. 441, no. 1, pp. 69-72, 2006.

[14] F.-R. F. Fan, J. Yang, L. Cai et al., "Charge transport through self-assembled monolayers of compounds of interest in molecular electronics," Journal of the American Chemical Society, vol. 124 , no. 19 , pp. 5550-5560, 2002. 
[15] F. Chen, X. Li, J. Hihath, Z. Huang, and N. Tao, "Effect of anchoring groups on single-molecule conductance: comparative study of thiol-, amine-, and carboxylic-acid-terminated molecules," Journal of the American Chemical Society, vol. 128, no. 49, pp. 15874-15881, 2006.

[16] E. Podstawka, Y. Ozaki, and L. M. Proniewicz, "Part III: surface-enhanced raman scattering of amino acids and their homodipeptide monolayers deposited onto colloidal gold surface," Applied Spectroscopy, vol. 59, no. 12, pp. 1516-1526, 2005.

[17] W. K. Paik, S. Han, W. Shin, and Y. Kim, "Adsorption of carboxylic acids on gold by anodic reaction," Langmuir, vol. 19, no. 10, pp. 4211-4216, 2003.

[18] L. A. Zotti, T. Kirchner, J. C. Cuevas et al., "Revealing the role of anchoring groups in the electrical conduction through single-molecule junctions," Small, vol. 6, no. 14, pp. 15291535, 2010.

[19] Y. Kim, T. J. Hellmuth, M. Bürkle, F. Pauly, and E. Scheer, "Characteristics of amine-ended and thiol-ended alkane single-molecule junctions revealed by inelastic electron tunneling spectroscopy," ACS Nano, vol. 5, no. 5, pp. 4104-4111, 2011.

[20] L. Venkataraman, J. E. Klare, C. Nuckolls, M. S. Hybertsen, and M. L. Steigerwald, "Dependence of single-molecule junction conductance on molecular conformation," Nature, vol. 442, no. 7105, pp. 904-907, 2006.

[21] L. Venkataraman, J. E. Klare, I. W. Tam, C. Nuckolls, M. S. Hybertsen, and M. L. Steigerwald, "Single-molecule circuits with well-defined molecular conductance," Nano Letters, vol. 6, no. 3, pp. 458-462, 2006.

[22] A. S. K. Hashmi and G. J. Hutchings, "Gold Catalysis," Angewandte Chemie, vol. 45, no. 47, pp. 7896-7936, 2006.

[23] D. J. Gorin and F. D. Toste, "Relativistic effects in homogeneous gold catalysis,” Nature, vol. 446, no. 7134, pp. 395-403, 2007.

[24] M.-C. Daniel and D. Astruc, "Gold nanoparticles: assembly, supramolecular chemistry, quantum-size-related properties, and applications toward biology, catalysis, and nanotechnology," Chemical Reviews, vol. 104, no. 1, pp. 293-346, 2004.

[25] M. D. Porter, T. B. Bright, D. L. Allara, and C. E. D. Chidsey, "Spontaneously organized molecular assemblies. 4. Structural characterization of $\mathrm{n}$-alkyl thiol monolayers on gold by optical ellipsometry, infrared spectroscopy, and electrochemistry," Journal of the American Chemical Society, vol. 109, no. 12, pp. 3559-3568, 1987.

[26] R. Yamada and K. Uosaki, "In situ scanning tunneling microscopy observation of the self-assembly process of alkanethiols on gold(111) in solution," Langmuir, vol. 14, no. 4, pp. 855-861, 1998.

[27] D. V. Leff, L. Brandt, and J. R. Heath, "Synthesis and characterization of hydrophobic, organically-soluble gold nanocrystals functionalized with primary amines," Langmuir, vol. 12, no. 20, pp. 4723-4730, 1996.

[28] S. Gomez, K. Philippot, V. Colliere, B. Chaudret, F. Senocq, and P. Lecante, "Gold nanoparticles from self-assembled gold(I) amine precursors," Chemical Communications, no. 19, pp. 1945-1946, 2000.

[29] M. Bardají, P. Uznanski, C. Amiens, B. Chaudret, and A. Laguna, "Aurophilic complexes as gold atom sources in organic media," Chemical Communications, no. 6, pp. 598599, 2002.
[30] W. W. Weare, S. M. Reed, M. G. Warner, and J. E. Hutchison, "Improved synthesis of small ( $\mathrm{dCORE} \approx 1.5 \mathrm{~nm}$ ) phosphinestabilized gold nanoparticies," Journal of the American Chemical Society, vol. 122, no. 51, pp. 12890-12891, 2000.

[31] M. Yamamoto and M. Nakamoto, "New type of monodispersed gold nanoparticles capped by myristate and $\mathrm{PPh}_{3}$ ligands prepared by controlled thermolysis of $\left[\mathrm{Au}\left(\mathrm{C}_{13} \mathrm{H}_{27} \mathrm{COO}\right)\left(\mathrm{PPh}_{3}\right)\right]$," Chemistry Letters, vol. 32, pp. 452-453, 2003.

[32] T. M. Owens, K. T. Nicholson, M. M. Banaszak Holl, and S. Süzer, "Formation of alkylsilane-based monolayers on gold," Journal of the American Chemical Society, vol. 124, no. 24, pp. 6800-6801, 2002.

[33] S. Watcharinyanon, D. Nilsson, E. Moons et al., "A spectroscopic study of self-assembled monolayer of porphyrin- functionalized oligo(phenyleneethynylene)s on gold: the influence of the anchor moiety," Physical Chemistry Chemical Physics, vol. 10, no. 34, pp. 5264-5275, 2008.

[34] E. Sabatani, I. Rubinstein, R. Maoz, and J. Sagiv, “Organized self-assembling monolayers on electrodes. Part I. Octadecyl derivatives on gold," Journal of Electroanalytical Chemistry, vol. 219, no. 1-2, pp. 365-371, 1987.

[35] L. G. Olson, Y. S. Lo, T. P. Beebe, and J. M. Harris, "Characterization of silane-modified immobilized gold colloids as a substrate for surface-enhanced Raman spectroscopy," Analytical Chemistry, vol. 73, no. 17, pp. 4268-4276, 2001.

[36] D. Briggs and M. P. Seah, Practical Surface Analyses, vol. 1, Wiley, New York, NY, USA, 2nd edition, 1990.

[37] T. A. Carlson and G. E. McGuire, "Study of the x-ray photoelectron spectrum of tungsten-tungsten oxide as a function of thickness of the surface oxide layer," Journal of Electron Spectroscopy and Related Phenomena, vol. 1, no. 2, pp. 161-168, 1972.

[38] B. R. Strohmeier, "ESCA method for determining the oxide thickness on aluminum alloys," Surface and Interface Analysis, vol. 15 , no. 1 , pp. $51-56,1990$.

[39] National Institute of Standards and Technology (NIST).

[40] M. A. Carvajal, J. J. Novoa, and S. Alvarez, "Choice of coordination number in d10 complexes of group 11 metals," Journal of the American Chemical Society, vol. 126, no. 5, pp. 1465-1477, 2004.

[41] E. Katzir, S. Yochelis, F. Zeides et al., "Increased superconducting transition temperature of a niobium thin film proximity coupled to gold nanoparticles using linking organic molecules," Physical Review Letters, vol. 108, no. 10, Article ID 107004, 5 pages, 2012.

[42] A. E. L. Wolf, Principles of Electron Tunneling Spectroscopy, Oxford University Press, New York, NY, USA, 1985.

[43] A. E. Hanna and M. Tinkham, "Variation of the Coulomb staircase in a two-junction system by fractional electron charge," Physical Review B, vol. 44, no. 11, pp. 5919-5922, 1991.

[44] H. H. Grabert and M. H. Devoret, Eds., Single Charge Tunneling, Plenum, New York, NY, USA, 1992.

[45] A. F. Andreev, "Thermal conductivity of the intermediate state of superconductors," Soviet physics, JETP, vol. 19, p. 1228, 1964.

[46] M. Tinkham, Introduction to Superconductivity, McGraw-Hill, New York, NY, USA, 1996. 

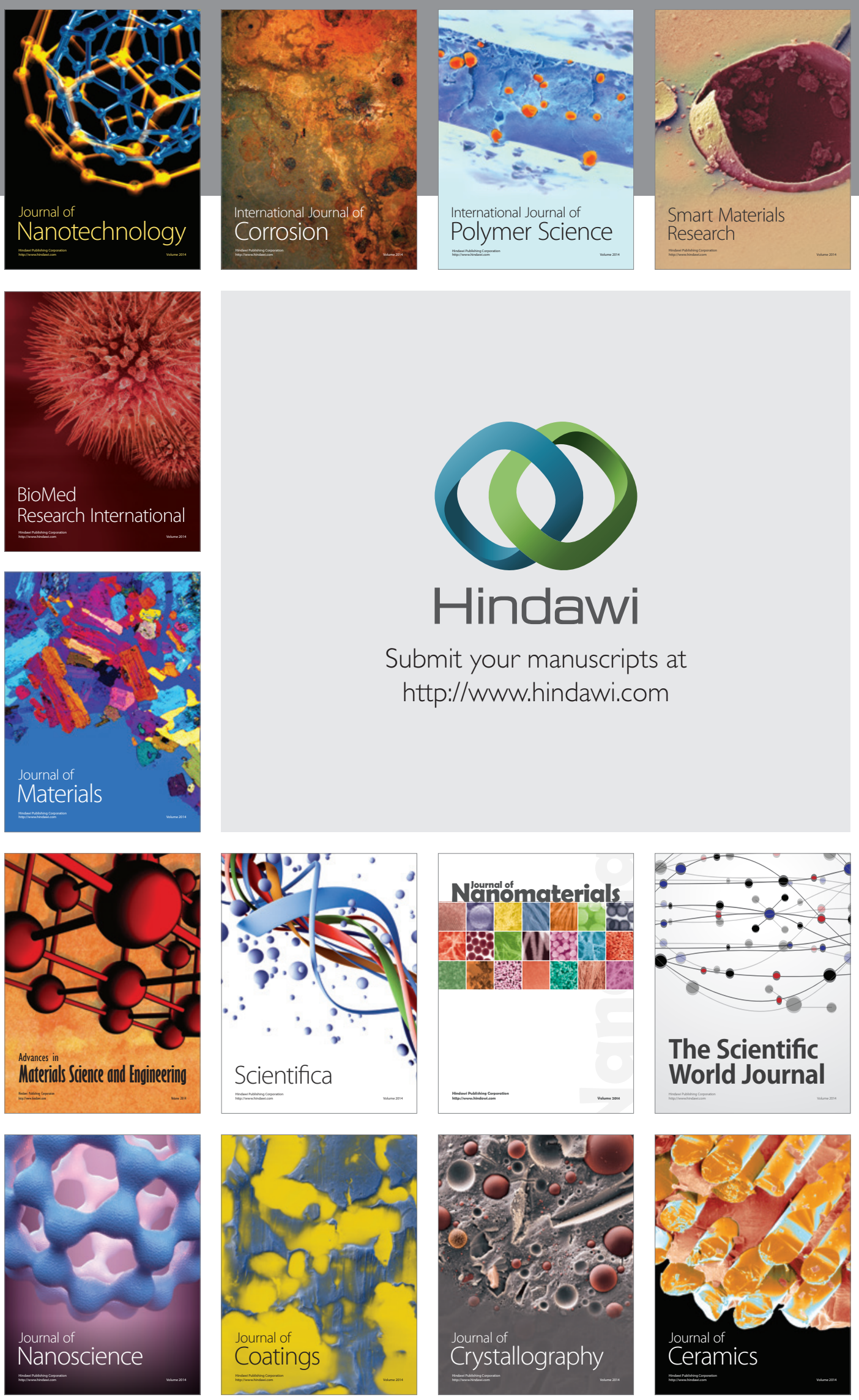

The Scientific World Journal

Submit your manuscripts at

http://www.hindawi.com

\section{World Journal}

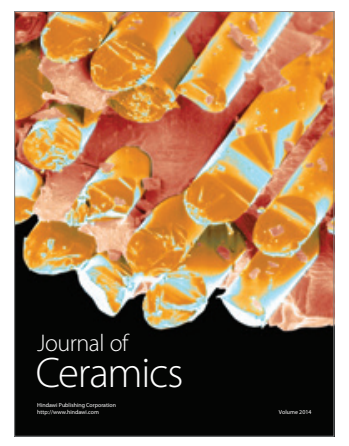

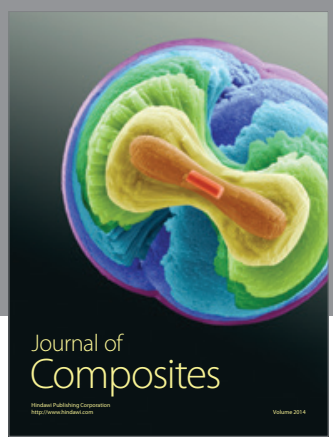
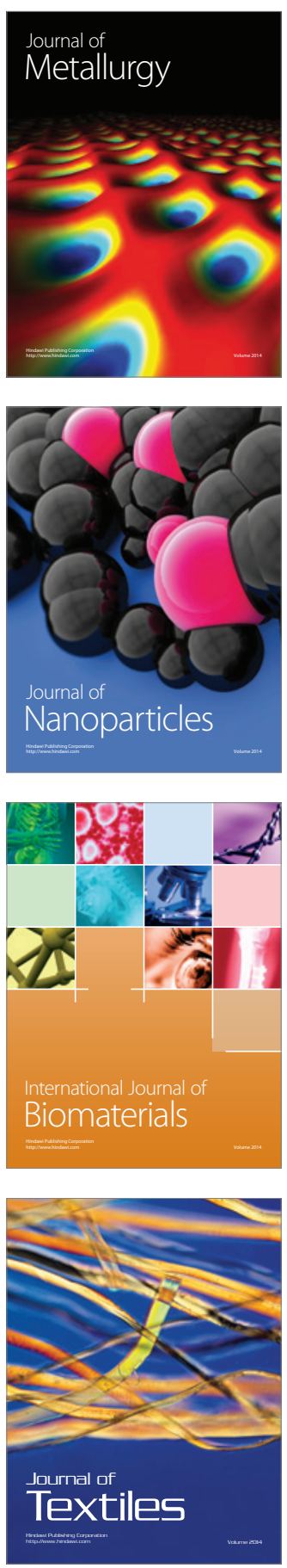